\title{
A Study on the Correlation between
}

\section{Writing Achievement and Reading Achievement of CSL Learners}

\author{
Li GUO \\ International Education Center \\ Beijing Language and Culture University \\ Beijing, China \\ hyguoli@163.com
}

\author{
Bo YAN \\ International Education Center \\ Beijing Language and Culture University \\ Beijing, China \\ yanbo12@blcu.edu.cn
}

\begin{abstract}
As the two crucial processes of output and input of language learning, writing and reading have been widely discussed by researchers. Aiming at giving suggestions on language acquisition for CSL learners, the author selects 865 essays from the HSK Dynamic Composition Corpus, examines them through Pearson correlation and discovers a significant mid-correlation between writing and reading. By dividing the essays into different writing ability groups, it is found that the level of writing does not make a great difference towards the correlation.
\end{abstract}

Keywords—correlation; writing achievement; reading achievement; CSL

\section{INTRODUCTION}

Relations between writing and reading have been investigated extensively over the past decades. Most researches reveal that writing and writing are highly correlated and share similar process. However, studies on the relationship of writing and read ing mainly involve English as a Second Language (ESL) learners and few studies study Chinese as a Second Language (CSL) learners' situation. Hence, this paper will explore the correlation between the writing achievement and the reading achievement of CSL learners. 865 essays named "Parents are the first teachers of the children" are selected from the HSK Dynamic Composition Corpus and Pearson correlation is applied to the whole samples first and then the samples of different writing ability groups to explore the influencing factor of the correlation.

\section{IITERATURE REVIEW}

Many researchers believe that writing activities would imply reading comprehension. On the contrary, through reading, writing abilities will also be effectively enhanced.

As early as 1980s, Woodford [1] compared the reading scores with the writing sores of "TOEIC" grades for 99 Japanese candidates and presented that the correlation between them reached 0.83 , which demonstrated that the reading scores greatly implies the writing scores. Collins \& Onwuegbuzie [2] examined the relationship between reading ability and writing proficiency among 115 African American graduate students enrolled in statistics courses. A canonical correlation analysis revealed that reading ability significantly predicted students' writing abilities. He argued that it is likely that reading ability plays a role in the technical writing process. Małgorzata Marzec-Stawiarska [3] showed a statistically significant difference in the reading skills of the two groups, suggesting that summarizing has a positive influence on reading development. Progress in students' reading skills did not correlate with the quality of the summaries they wrote. Juhee Lee and Diane L. Schallert [4] suggest that the reading-writing connection seems more evident for those second-language learners whose language proficiency exceeds a certain linguistic threshold for writing.

The research of Chinese scholars mainly focuses on the study of the correlation between English reading and writing. Through the reading scores and the writing scores of the subjects, the correlation coefficient between the two is analyzed, and further concludes the influence between them. Due to the different subjects and means of testing, the correlations obtained from the researchers also vary. Zeng Xiangmin [5] conducted a survey and the correlation coefficient between English reading and writing was 0.37. Tan Chengqiang [6] argued that reading activities and writing activities are very complex processes. Moreover, the improvement of English writing ability depends on a series of interrelated, mutual-influence factors, which includes reading ability. Wang yingying [7] extracted the reading scores and writing scores of CET 4 as the research samples and declaimed that the correlation coefficient was 0.42 . Zhang Yunqing [8] studied the relationship between reading and writing, and the results showed that there was a positive correlation between English reading ability and writing ability of English major students. Whereas, some 
research assumes that writing achievement and reading achievement of second language learners bears little relationship. Xu Feng [9] studied the correlation between the receptive skills and the productive skills, showing that their coefficient is very low (0.183).

However, little research involved with Chinese as the second language learners (CSL) is conducted. Research on CSL is mainly constricted to the lexical domain. Wang Yixuan [10] takes lexical diversity, lexical sophistication, lexical frequency profile and lexical errors from the framework as measuring dimensions, basing on 360 sample compositions from the HSK Dynamic Corpus of Writing Paper, the results show that lexical errors, the number of types and frequency words are effective parameters for writing quality. Hong Wei and Chen Nan [11] focus on how Chinese as a second language (CSL) learners differentiate between the various meanings of Chinese near-synonyms. Via multiple-choice tests, self-assessment of learning difficulty and interviews, they found that CSL learners perform diversely in similar senses as well as dissimilar senses at the primary stage, the intermediate stage and the advanced stage. The findings indicated that the near-synonymous construction pattern in the internal lexicon goes through a dynamic changing process from word-based connection to word-sense-based connection. In conclusion, the research on the correlation between reading performance and writing performance of the CSL learners is seldom conducted.

\section{RESEARCH DESIGN} Corpus.

This paper extracts 865 essays named "Parents are the first teachers of the children" from the HSK Dynamic Composition

The author employs the method of Pearson correlation analysis to explore the relationship between the Chinese essay achievements and the reading achievements of the candidates. Furthermore, the candidates are divided into 4 groups (Group A $\geq 90$, $80 \leqq$ Group $\mathrm{B}<90,60 \leqq$ Group $\mathrm{C}<80$, Group $\mathrm{D}<60$ ) according to the scores from high to low. And then the correlation between the writing achievements and the reading achievements of the candidates are investigated respectively.

\section{DATA ANALYSiS}

This paper employs SPSS 22.0 to explore the correlation between writing achievement and reading achievement of CSL learners. The two variables, the writing scores and the reading scores of the CSL learners, are measured by Pearson correlation to look into if there is any liner correlation between them.

\section{A. The correlation between the writing scores and the reading scores of 865 CSL learners}

TABLE I. THE CORRELATION BETWEEN THE WRITING SCORES AND THE READING SCORES OF 865 CSL LEARNERS

\begin{tabular}{|l|l|l|l|}
\hline \multirow{3}{*}{ Writing Scores } & & \multicolumn{1}{|c|}{ Writing Scores } & \multicolumn{1}{|c|}{ Reading Scores } \\
\hline & Pearson Correlation & 1 & $.432 * *$ \\
\cline { 2 - 4 } & Significance (two-tailed) & & .000 \\
\cline { 2 - 4 } & $\mathrm{N}$ & 865 & 865 \\
\hline
\end{tabular}

The paper first examines the correlation between the writing scores and the reading scores of the 865 CSL learners. The P value is $0.00<0.5$, and the Pearson correlation coefficient is 0.432 , implying a mid-correlation between them.

\section{B. The correlation between the writing scores and the reading scores in different writing ability groups}

The correlation between the writing scores and the reading scores in different writing ability groups is further investigated. The candidates are divided into 4 groups according to their writing scores from the HSK Dynamic Composition Corpus. Then Pearson correlation is utilized to expound the relationship between the writing achievement and reading achievement of the candidates from different writing ability groups.

TABLE II. THE CORRELATION BETWEEN THE WRITING SCORES AND THE READING SCORES OF GROUP A

\begin{tabular}{|l|l|l|l|}
\hline \multirow{3}{*}{ Writing Scores } & & Writing Scores & \multicolumn{1}{|c|}{ Reading Scores } \\
\hline & Pearson Correlation & 1 & $.349^{* *}$ \\
\cline { 2 - 4 } & Significance (two-tailed) & & .003 \\
\cline { 2 - 4 } & $\mathrm{N}$ & 68 & 68 \\
\hline
\end{tabular}

68 candidates are classified into Group A. The correlation between the writing scores and reading scores of the candidates in Group A is significantly correlated with a low correlation coefficient 0.349 . $(\mathrm{P}=0.003<0.05)$ 
TABLE III. THE CORRELATION BETWEEN THE WRITING SCORES AND THE READING SCORES OF GROUP B

\begin{tabular}{|l|l|l|l|}
\hline & & \multicolumn{1}{|c|}{ Writing Scores } & \multicolumn{1}{c|}{ Reading Scores } \\
\hline \multirow{4}{*}{ Writing Scores } & Pearson Correlation & 1 & -.004 \\
\cline { 2 - 4 } & Significance (two-tailed) & & .966 \\
\cline { 2 - 4 } & $\mathrm{N}$ & 111 & 111 \\
\hline
\end{tabular}

Group B covers 111 candidates. The $\mathrm{P}$ value of Pearson correlation between the writing scores and reading scores of the candidates in Group B is $0.966>0.05$, implying no significant difference. It is worthy to note that the correlation coefficient is negative (-0.004), which indicates that the candidates with middle-level writing skills confront such a bottleneck period that their writing achievement may not stimulate the reading achievement or vice versa.

TABLE IV. THE CORRELATION BETWEEN THE WRITING SCORES AND THE READING SCORES OF GROUP C

\begin{tabular}{|l|l|l|l|}
\hline & & \multicolumn{1}{|c|}{ Writing Scores } & \multicolumn{1}{c|}{ Reading Scores } \\
\hline \multirow{4}{*}{ Writing Scores } & Pearson Correlation & 1 & $.244^{* *}$ \\
\cline { 2 - 4 } & Significance (two-tailed) & & .000 \\
\cline { 2 - 4 } & $\mathrm{N}$ & 631 & 631 \\
\hline
\end{tabular}

631 candidates obtain a score in the range of 60 to 80 . The Pearson correlation coefficient between the writing scores and reading scores of the candidates in Group $\mathrm{C}$ is 0.244 with a $\mathrm{P}$ value of $0.00(\mathrm{P}<0.05)$, indicating a significant difference between them, though the correlation coefficient is relatively low.

TABLE V. THE CORRELATION BETWEEN THE WRITING SCORES AND THE READING SCORES OF GROUP D

\begin{tabular}{|l|l|l|l|}
\hline \multirow{3}{*}{ Writing Scores } & & \multicolumn{1}{|c|}{ Writing Scores } & \multicolumn{1}{c|}{ Reading Scores } \\
\hline & Pearson Correlation & 1 & $.170^{*}$ \\
\cline { 2 - 4 } & Significance (two-tailed) & & .029 \\
\cline { 2 - 4 } & $\mathrm{N}$ & 166 & 166 \\
\hline
\end{tabular}

Group D includes the candidates with an inferior writing ability. The correlation between the writing scores and reading scores of the 166 candidates in Group D shows a significant difference. The correlation coefficient is 0.170 and the $\mathrm{P}$ value is 0.029 $(<0.05)$.

\section{DISCUSSION}

The results reveal that on the whole, the correlation between the writing scores and the reading scores of 865 CSL learners is significantly correlated with a coefficient of 0.432 . The writing achievement demonstrates a predictive effect for their reading achievement, for writing helps CSL learners promote the use of the syntax structure and the words of target language and facilitate the automation of the language use, thus effectively achieving the purpose of language acquisition. CSL learners' language skills are continuing to be consolidated and internalization via writing, paving the way for the overall development of language skills. Just as Swain's Output Hypothesis states, output is crucial for language acquisition and learners need the opportunities to use linguistic resources for the sake of achieving full grammatical competence. Extensive output practice may improve the input skills.

At the same time, Krashen [12] argues that comprehensive input is the driving force for interlanguage development and change, and that effects of such change carry over to influence production, which means high receptive skills will also spontaneously lead to high-level productive skills. In this view, output is identically significant as a device to negotiate better input: input would also be a major explanatory construct. Therefore, the output and the input are interactive and have different functions in language learning or acquisition. In the case of reading and writing, the reading ability enriches the CSL learners' schema, while the writing ability reinforces the knowledge they have already had in the schema, and tests the knowledge to achieve communitive goals successfully and effectively. These two abilities usually present mutual effects on each other. Hence, a good writer often has a better understanding of the reading materials; similarly, extensive reading practice may stimulate the learners' writing potentials.

The results of the four groups show that the degree of the correlation tends to be enhanced as the scores rise from low to high, except Group B. Group B is the only group that the $\mathrm{P}$ value is not significant and the correlation coefficient is negative. The reasons may be that the learners in this domain confront the bottleneck of language learning. The candidates with middle and upper level writing ability may not have an equivalent reading ability. The writing achievement and the reading achievement of middle-level learners do not show significant correlation. 
It is remarkable that although the $\mathrm{P}$ values of the four groups are below 0.05 , which represent a significant correlation between the writing scores and reading scores of the CSL learners, the Pearson correlation coefficient is low (<0.4). This could be resulted from the interference of the rating system. Some learners could write essays with complex sentence patterns and graceful expressions, but it is hard for them to guarantee the accuracy of the sentences, which may bear more grammatical errors or wrongly written words. In HSK rating system, these mistakes would decrease the scoring grades to some extent. Otherwise, some learners can only express themselves by simple sentences and characters, greatly reducing the frequency of the mistakes instead, and obtain relatively satisfactory scores. Therefore, the writing scores do not authentically reflect the learners' writing ability, which leads to interference towards the correlation between the writing achievement and the reading achievement of the learners.

Another reason why the degree of the correlation is low may lie in the fact that writing and reading are two relatively independent abilities after all. The main process of reading involves acquiring the comprehension through the reader's own language knowledge, grammar knowledge, genre knowledge, etc. The reading achievements are limited by the reading materials. Whereas, the writing process is to form a new discourse by using organizational capacity based on the ideation. Those who are free to express their thoughts do not necessarily understand the views of others. The high level of writing implies relatively high organizational capacity and ideation, but not necessarily a high understanding of the discourse, thus not indicating a high level of reading. Therefore, a "good writer" is not necessarily a "good reader" and a "good reader" is not necessarily a "good writer" either.

\section{SUMMARY}

This paper investigates the correlation between the writing achievement and reading achievement of CSL learners based on the HSK Dynamic Composition Corpus and asserts that they have a significant mid-correlation. In addition, the level of writing does not make a great difference towards the correlation between the writing achievement and the reading achievement of CSL learners. The conclusion has a great effect on second language acquisition. Reading and writing share "common language knowledge basis and empirical schema foundation" and interact with each other. Reading increases writing knowledge and provide "comprehensible input" for writing. Meanwhile, writing also promote reading ability, playing a positive role in return. Many research findings show that the difficulties of writers are actually lacking in the expression of vocabulary, and it is much easier for learners with strong reading ability to get expressions from the reading materials, and thus having more abundant vocabulary in essay writing. As a consequence, reading and writing should be treated jointly to achieve a multiplier effect in second language acquisition.

\section{ACKNOWLEDGMENT}

The paper was prepared with support of Science Foundation of Beijing Language and Culture University (supported by “the Fundamental Research Funds for the Central Universities").

\section{REFERENCES}

[1] E.P. Woodford, An Introduction to TOEIC: the Initial Validity Study. Educational Testing Service, 1982.

[2] K.M.T. Collins and A.J. Onwuegbuzie, "Reading ability as a predictor of African-American graduate students' writing proficiency in the context of statistics courses" in Journal of Negro Education, vol. 83, 2014, pp.135-138.

[3] M. Marzec-Stawiarska, "The influence of summary writing on the development of reading skills in a foreign language" in System, vol. 59, 2016, pp.90-100.

[4] J. Lee and D.L. Schallert, "Exploring the reading-writing connection: a yearlong classroom-based experimental study of middle school students developmenting literacy in a new language" in Reading Research Quarterly, vol. 51, NJ: Wiley-Blackwell, 2016, pp.143-165.

[5] X.M. Zeng and C.H. Lu, "The correlation between EFL reading comprehension ability and writing ability of Chinese college students" in Journal of Sichuan College of Education, vol. 21, 2005, pp.62-65.

[6] C.Q. Qin and L.Y. Huang, "The relationship between English reading ability and writing ability" in Journal of Guangxi University ( Philosophy and Social Science), vol. 29, 2007, pp.79-82.

[7] Y.Y. Wang, "Research on the correlation between reading comprehension and writing in CET-4" in Journal of Hunan First Normal College, vol. 8, 2008, pp.69-71.

[8] Y.Q. Zhang, L. Zhang and Z.C. Fan, "The correlation between English reading ability and writing ability" in Journal of Hunan University of Science and Engineering, vol. 31, 2010, pp. 163-165.

[9] F. Xu, "Study on the correlation between receptive skills and productive skills" in Foreign Language Teaching and Research, vol. 36, Beijing: Foreign Language Teaching and Research Press, 2003, pp.80-82.

[10] Y.X. Wang, "The correlation between lexical richness and writing score of CSL learner-the multivariable linear regression model and equation of writing quality" in Applied Linguistics, 2017, pp.93-101.

[11] W. Hong and N. Chen, "A study on the L2 acquisition of differences in similar sense and dissimilar of sense of Chinese near-synonyms" in Applied Linguistics, 2013, pp.99-106.

[12] S.D. Krashen, Principles and Practice in Second Language Acquisition, American: Pergamon Press, 1982. 